\title{
Reduction in alcohol consumption and health status
}

\author{
Wenbin Liang \& Tanya Chikritzhs \\ National Drug Research Institute, Curtin University of Technology, Perth, Western Australia, Australia
}

\begin{abstract}
Aims This study investigated the association between alcohol consumption and health status using cross-sectional national survey data. Measurements and design This study relied upon self-report data collected by the 2004 and 2007 Australian National Drug Strategy Household (NDSH) surveys. Households were selected using a multi-stage, stratified-area, random sample design. Both surveys used combinations of the drop-and-collect and computer-assisted telephone interview approaches. Respondents were questioned about their current and past drinking, the presence of formal diagnosis for specific diseases (heart disease, type 2 diabetes, hypertension, cancer, anxiety, depression) and self-perceived general health status. Associations between drinking status, the presence of diagnoses and selfperceptions of general health status among respondents aged $18+$ and $45+$ were assessed using multivariate logistic regression. Setting and participants Males and females aged 18 years or older and resident in Australia. The sample sizes for the 2004 and 2007 NDSH surveys were 24109 and 23 356, respectively. Findings Respondents with a diagnosis of diabetes, hypertension and anxiety were more likely to have reduced or stopped alcohol consumption in the past 12 months. The likelihood of having reduced or ceased alcohol consumption in the past 12 months increased as perceived general health status declined from excellent to poor. Conclusions Experience of ill health is associated with subsequent reduction or cessation of alcohol consumption. This may at least partly underlie the observed 'J-shape' function relating alcohol consumption to premature mortality.
\end{abstract}

Keywords Alcohol, Australia, chronic disease, epidemiology, general population.

Correspondence to: Wenbin Liang, National Drug Research Institute, Curtin University of Technology, Perth, Western Australia, Australia.

E-mail: w.liang@curtin.edu.au

Submitted 12 May 2010; initial review completed 1 July 2010; final version accepted 14 July 2010

\section{INTRODUCTION}

During several decades, many cohort studies from the medical epidemiology literature have observed a 'J-shaped' association between alcohol consumption and risk of coronary heart disease (CHD) and stroke for people aged in their middle years and older [1-4]. Non-drinkers thereby appear to have a higher risk of $\mathrm{CHD}$ and stroke compared to those who consume alcohol regularly at moderate levels. The direct implication of the J-shaped curve is that moderate alcohol consumption 'protects' against cardiovascular disease. The veracity of the J-shaped curve has been questioned increasingly in recent years [5-10]. Naimi et al. showed that nondrinkers had higher prevalence of risk factors for CHD and stroke, and that the 'protective' effect of moderate alcohol consumption may well be due to residual and unmeasured confounding factors [11]. The association between non-drinkers and poor general health status was also observed by Baumeister et al., who showed a higher rate of physician visits compared to moderate drinkers [12]. Indeed, the so-called 'sick-quitter' hypothesis proposes that a large proportion of non-drinkers quit drinking as they age and develop chronic disease conditions [13]. There are two Australian studies which have investigated the effect of health status on alcohol consumption behaviours: one among a sample of 45-50-year-old women [14] and another using a sample of 18-59-yearold Canberra residents [15]. Both studies suggested that poor health led to reduction in alcohol consumption $[14,15]$, and their findings need to be confirmed further by a representative national sample study.

The 2004 and 2007 Australian National Drug Strategy Household (NDSH) surveys obtained information on changes in alcohol consumption and disease conditions. Using a validated questionnaire, health status and demographic characteristics were self-reported from a large random sample of the general Australian population 
aged 12 years and older. The aim of this study was to investigate the association between changes in alcohol consumption and health status using cross-sectional data drawn from these two national surveys. We included two types of measures of health status: (1) diagnosis of chronic diseases and (2) self-perceived health status.

\section{METHOD}

\section{Data source}

Data collected during the 2004 and 2007 NDSH surveys were combined and analysed together. Details of sampling strategy and data collection methods have been described in the 2007 NDSH survey [16]. Briefly, the NDSH survey is a national survey targeting the $12+$ years Australian population. Both the $2004(n=24$ 109) and 2007 ( $n=23$ 356) surveys used combinations of the drop-and-collect and the computer-assisted telephone interview (CATI) approaches to collect information from household respondents. Households were selected by a multi-stage, stratified-area, random sample design. Both surveys asked the same detailed questions about respondent alcohol consumption history and changes to alcohol consumption in the last 12 months. Both survey questionnaires can be obtained from the Australian Institute of Health and Welfare website at http://www. aihw.gov.au.

\section{Drinking groups}

Respondents who answered 'no' to the question: 'Have you ever had a full serve of alcohol, for example, a glass of wine, a whole nip of spirits, a glass of beer, etc.', were classified as 'life-long abstainers'.

In keeping with the most recent Australian National Health and Medical Research Council drinking guidelines [17], current 'moderate drinkers' were defined as respondents who reported usually drinking one to two standard drinks (10 g pure alcohol) per day in the quantity/ frequency component of the survey over the past 12 months. Current drinkers who exceeded one to two standard drinks per day were referred to as 'non-moderate drinkers'. Moderate and non-moderate drinkers combined were referred to as 'current drinkers'.

Respondents who reduced their alcohol consumption in the last 12 months were identified as those who answered 'yes' to having had a drink in the past 12 months but who also answered 'yes' to any of the following options: 'In the last 12 months have you . . . reduced the amount of alcohol you drink at any one time and/or reduced the number of times you drink and/or switched to drinking more low-alcoholic drinks than you used to and/or stopped drinking alcohol?'. Respondents who were currently drinking but who had reduced their consumption in the past 12 months were referred to as 'recent reducers'. Current drinkers who had not reduced their consumption in the past 12 months were referred to as 'stable current drinkers'.

Respondents who answered 'no' to the question: 'Have you had an alcoholic drink of any kind in the last twelve months?' but who answered 'yes' to: 'Have you ever had a full serve of alcohol?' were classified as 'ex-drinkers (1 year + )'. Although currently non-drinkers, these respondents were not life-time abstainers; they may, however, have stopped consumption more than 12 months ago. The NDSH survey questions do not allow estimation of the length of non-drinking time beyond 12 months.

\section{Health status}

Questions in the 2004 and 2007 NDSH surveys about current health status included: (i) whether or not the respondent had received a diagnosis and/or treatment for one or more (i.e. multiple positive responses were possible) specifically listed chronic diseases in the 12 months prior to the survey; and (ii) self-ranked general health status (excellent, very good, good, fair, poor) for health status at the time of the survey. The specific chronic diseases (prevalence among 18 years+ sample) included: diabetes (5.4\%); heart disease (5.8\%); hypertension (17.8\%); cancer (3.1\%); depression (9.0\%); and anxiety $(4.6 \%)$.

\section{Data analysis}

Multivariate analysis included subjects older than 18+ years with analysis repeated for the $45+$ years population (reporting of J-shaped curves have typically been limited to the 45+-year age group). Separate logistic regression models were employed to:

1 investigate whether there was an association between reduced alcohol consumption and health status in the last 12 months prior to survey;

2 investigate whether there was an association between non-life-time alcohol abstention (recent reducers) and health status in the last 12 months; and

3 compare the association between self-reported health status and volume of usual alcohol consumption over the last 12 months prior to the survey among the 45 years+ subjects to the 'J-shaped' association observed in epidemiology studies.

All models adjusted for a range of potential cofounders including: age, sex, state of residency, income level, highest level of education attained, indigenous status, tobacco use (ever) and marital status. These confounders were measured and related to the time of the survey. We note that few cohort studies which have concluded protective effects of moderate alcohol on various types of chronic disease have controlled for marital status as a 
potential confounder [18]. Only respondents who answered all questions relating to all variables in the respective models were included in analyses.

\section{RESULTS}

\section{Recent reducers versus non-reducers}

Table 1 shows logistic regression results testing the association between chronic disease and whether or not alcohol consumption was reduced during the last 12 months. Respondents aged 18 years+ with a diagnosis of diabetes were $40 \%$ more likely to report having reduced their alcohol consumption. Similarly, significant positive associations were observed between reduced alcohol consumption and diagnoses of hypertension and anxiety. The observed associations were similar when limited to those aged 45 years or older. Although the odds ratios (OR) failed to reach significance, respondents aged 45 years or older who had received a diagnosis of heart disease were less likely to reduce alcohol consumption in the last 12 months.

Table 1 also shows the association between selfreported general health status and reduced alcohol consumption in the last 12 months. A positive linear trend was observed, suggesting that people with worse general health status were more likely to reduce their alcohol consumption. Moreover, the ORs increased by about 20\% after limiting the analysis to the population aged 45 years and older, i.e. those in middle age or older appeared to have greater odds of reducing their alcohol consumption with declining general health status (although the confidence intervals for $18+$ and $45+$ overlap).

\section{Ex-drinkers (1 year + ) versus current drinkers}

Evidence for significant associations between diagnoses of specific chronic disease and the likelihood of having stopped drinking in the last 12 months was limited to diabetes (Table 1). However, a positive linear association was found for self-reported general health status and the likelihood of being an ex-drinker for 1 year or longer. That is, people with worse perceived general health status were more likely to have ceased alcohol consumption at some time before the last 12 months (Table 1).

\section{Level of alcohol consumed and perceived general health status}

Table 2 shows that being a recent abstainer, a life-long abstainer or drinking five or more drinks per day was associated with worse perceived general health status over the last 12 months. This is in keeping with the observation from other studies that people with poor health and health problems are likely to reduce or stop their alcohol consumption [18].

\section{DISCUSSION}

In this study, it was observed that people with a diagnosis of diabetes, hypertension and anxiety were more likely to reduce or stop alcohol consumption in the past 12 months. Similarly, the likelihood of having reduced or ceased alcohol consumption in the past 12 months increased as perceived general health status declined from excellent to poor. Our results are consistent with previous findings by Power et al. [14] and Rodgers et al. [15].

Self-perceptions of health status may be related more strongly to changes in drinking status than the presence or absence of formal diagnosis. For example, prior to onset of obvious symptoms, a person with an undiagnosed chronic condition (e.g. diabetes, liver cirrhosis, cancer-not necessarily illness related directly to alcohol use) may subjectively 'feel well' and be unlikely to alter their drinking status on this basis. However, when symptoms begin to appear, the physical manifestations of ill-health may precipitate behaviour change, including reduced alcohol consumption [13]. Furthermore, having been prescribed medication or treated for chronic disease may influence an individual's perception of their own health status. This would concur with our observation that there was a significant association between having received treatment for at least one health condition over the last 12 months and selfperceived health status.

The potential effects of mental health disorders on alcohol use should also not be overlooked, as they have been found to be a contributing cause for many types of chronic diseases including hypertension, heart diseases and cancers [19]. It has been suggested by previous studies that self-perception of health is linked to symptoms of mental health disorders and other chronic illnesses [20,21]. A large proportion of mental health disorders remain undiagnosed, and therefore using self-perception of health status may be more useful for predicting changes in drinking status or accounting for potential health-status-related confounding than the presence of formal diagnosis.

These results are in keeping with other research evidence, which suggests that drinkers tend to reduce or stop their alcohol consumption when faced with declining health status [6,22]. Although reverse causality cannot be entirely ruled out, given the cross-sectional design of this study, it is unlikely that having reduced or ceased alcohol consumption in the past 12 months leads to the development of declining general heath, hypertension or anxiety. 


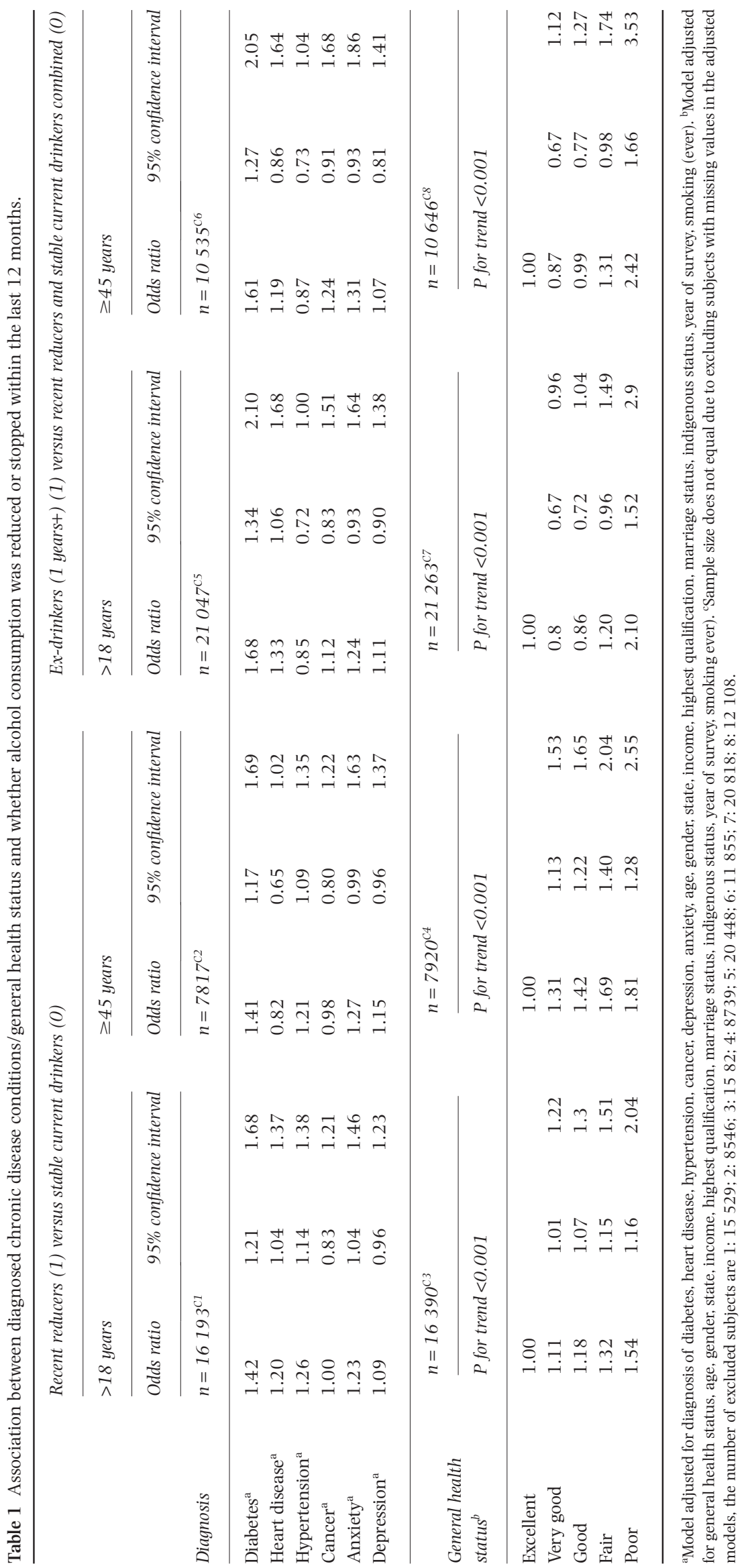


Table 2 Alcohol consumption level and health status having fair and poor health as positive (1) outcome versus having good, very good and excellent health (0) among subjects 45 years $+^{\mathrm{a}}$.

\begin{tabular}{llll}
\hline Usual alcohol consumption & Odds ratio & \multicolumn{2}{c}{ 95\% confidence interval } \\
\hline 13 or more drinks & 3.77 & 2.48 & 5.74 \\
11-12 drinks & 3.03 & 2.01 & 4.57 \\
$7-10$ drinks & 1.49 & 1.20 & 1.86 \\
5-6 drinks & 1.33 & 1.14 & 1.56 \\
3-4 drinks & 1.03 & 0.92 & 1.15 \\
1-2 drinks & 1.00 & & \\
Abstainer last 12 months & 1.85 & 1.63 & 2.09 \\
Life-long abstainer & 1.33 & 1.07 & 1.66 \\
\end{tabular}

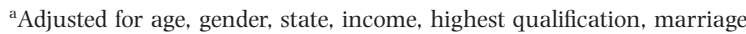
status, indigenous status, year of survey, smoking (ever).

The development of hypertension, for instance, is an ongoing long-term process which may start in childhood [23], and reduced alcohol consumption in the past 12 months is very unlikely to 'cause' hypertension in respondents. Moreover, despite the fact that the prevalence of undiagnosed type 2 diabetes mellitus is likely to be high in Australia, screening studies suggest that the average latency period for diagnosis is at least 1.5 years [24]. Thus, in the vast majority of cases considered here, it is reasonable to assume that the development of type 2 diabetes and its eventual diagnosis occurred well in advance of changes to alcohol consumption which may have occurred in the past 12 months.

Both diabetes and hypertension are well-recognized risk factors for CHD and stroke [25], and there is strong evidence to suggest that anxiety is associated with an increased risk of CHD [26]. Thus, the results presented here support the hypothesis that drinkers 'quit' or reduce drinking because of chronic disease conditions which increase in frequency throughout the life-span [6,22] and concur with the observations made by others that 'non-drinkers' have a higher prevalence of risk factors for CHD and stroke [11].

The phenomenon of the 'sick quitter' may be viewed as similar to the loss of subjects to clinic trials. Subjects who begin as 'drinkers' but who ultimately stop drinking while participating in a cohort study are analogous to subjects in clinical trials who drop out of treatment for reasons which are related to the prognosis itself. In clinical trials, subject dropout potentially introduces a bias, because people who complete a particular treatment may, at the outset, be predisposed to have a better outcome $[27,28]$. In the same way, people who do not become ex-drinkers may be predisposed to have better health outcomes.

In order to reduce bias in clinical trials, 'intention-totreat' analysis is recommended [28]. This essentially involves 'returning' any subjects who had withdrawn from the trial along with their health outcomes back into the group to which they had originally been assigned. This mitigates any false impression of low morbidity or mortality which may arise from not including adverse outcomes for subjects who withdrew from a treatment group, and thereby maintains the integrity of the randomization process. In the same way, the health profiles of people who cease drinking (i.e. treatment) at some point in a cohort study should not be considered to form a new group of 'ex-drinkers', but should be 'returned' to the drinker group to which they were originally assigned on the basis of their alcohol consumption.

\section{Limitations}

This study relies upon a cross-sectional design and measures self-reported alcohol consumption and health status. It has been well documented that surveys almost always underestimate total alcohol consumption [29]. It has been estimated that, on average, the level of underestimation across the national Australian population is about $28 \%$. This implies that in reality, the proportion of drinkers who consume at low risk levels in relation to the NDSH survey guidelines is an overestimate, as a proportion of such respondents actually drink at risky levels [29]. How this underestimation would influence, if at all, the relation between alcohol consumption and health status demonstrated here is not clear and requires further investigation.

The response rates were $49.3 \%$ for the 2007 survey and $46 \%$ for the 2004 survey. Despite the large national samples attained and the representativeness of the sampling frame, with surveys of this nature it is possible that the proportion of the approached sample which agrees to participate in a health study is different from the population which refuses. This study therefore provides evidence of an association between change of alcohol consumption and health status within a self-referred health study population. Arguably, populations with similar propensities or characteristics are also more likely to participate in and respond to other survey-based studies. A large number of subjects with missing values in controlled variables were excluded in the multivariate logistic regression, and this may have introduced systematic error. Logistic models controlling only for diagnosis of disease/general health status, sex and age were, however, compared with the full models. Similar estimations were observed, suggesting that systematic error due to exclusion of subjects with missing values was unlikely.

These surveys were conducted on an Australian sample, and human behaviours may vary by country and culture. Nevertheless, findings drawn from these surveys are consistent with those from studies conducted in at least two other western nations: Germany [12] and the United States $[11,13]$. 
NDSH survey respondents self-reported their diagnoses of chronic disease. As there is no medical examination to confirm these self-reports their accuracy is unknown; however, there is no reason to suspect that substantial numbers of respondents would have cause to falsify or be mistaken about their diagnoses.

\section{CONCLUSION}

Respondents with a diagnosis of diabetes, hypertension and anxiety were more likely to have reduced or stopped alcohol consumption in the past 12 months. The likelihood of having reduced or ceased alcohol consumption in the past 12 months increased as perceived general health status declined from excellent to poor. Experience of ill health is associated with subsequent reduction or cessation of alcohol consumption. This may at least partly underlie the observed J-shaped function relating alcohol consumption to premature mortality.

In addition to questions regarding the presence of formal diagnoses for major health conditions, cohort studies which seek to examine the relation between alcohol and chronic disease should question participants on their perceived level of general physical and mental health at outset and follow-up.

\section{Declarations of interest}

None.

\section{References}

1. Corrao G., Rubbiati L., Bagnardi V., Zambon A., Poikolainen K. Alcohol and coronary heart disease: a meta-analysis. Addiction 2000; 95: 1505-23.

2. Reynolds K., Lewis L. B., Nolen J. D. L., Kinney G. L., Sathya B., He J. Alcohol consumption and risk of stroke: a meta-analysis. JAMA 2003; 289: 579-88.

3. Rimm E. B., Williams P., Fosher K., Criqui M., Stampfer M. J. Moderate alcohol intake and lower risk of coronary heart disease: meta-analysis of effects on lipids and haemostatic factors. BMJ 1999; 319: 1523-8.

4. Wulsin L. R., Singal B. M. Do depressive symptoms increase the risk for the onset of coronary disease? A systematic quantitative review. Psychosom Med 2003; 65: 201-10.

5. Shaper A. G., Wannamethee G., Walker M. Alcohol and mortality in British men: explaining the U-shaped curve. Lancet 1988; 332: 1267-73.

6. Wannamethee G., Shaper A. G. Changes in drinking habits in middle-aged British men. J R Coll Gen Pract 1988; 38: 440-2.

7. Fillmore K. M., Stockwell T., Chikritzhs T., Bostrom A., Kerr W. Moderate alcohol use and reduced mortality risk: systematic error in prospective studies and new hypotheses. Ann Epidemiol 2007; 17: S16-23.

8. Chikritzhs T., Fillmore K., Stockwell T. I. M. A healthy dose of scepticism: four good reasons to think again about protective effects of alcohol on coronary heart disease. Drug Alcohol Rev 2009; 28: 441-4.
9. Jackson R., Broad J., Connor J., Wells S. Alcohol and ischaemic heart disease: probably no free lunch. Lancet 2005; 366: 1911-12.

10. World Health Organization. Prevention of Cardiovascular Disease: Guidelines for Assessment and Management of Cardiovascular Risk. Geneva: World Health Organization; 2007.

11. Naimi T. S., Brown D. W., Brewer R. D., Giles W. H., Mensah G., Serdula M. K. et al. Cardiovascular risk factors and confounders among nondrinking and moderate-drinking U.S. adults. Am J Prev Med 2005; 28: 369-73.

12. Baumeister S. E., Schumann A., Nakazono T. T., Alte D., Friedrich N., John U. et al. Alcohol consumption and out-patient services utilization by abstainers and drinkers. Addiction 2006; 101: 1285-91.

13. Rehm J., Irving H., Ye Y., Kerr W. C., Bond J., Greenfield T. K. Are lifetime abstainers the best control group in alcohol epidemiology? On the stability and validity of reported lifetime abstention. Am J Epidemiol 2008; 168: 866-71.

14. Powers J. R., Young A. F. Longitudinal analysis of alcohol consumption and health of middle-aged women in Australia. Addiction 2008; 103: 424-32.

15. Rodgers B., Korten A. E., Jorm A. F., Christensen H., Henderson S., Jacomb P. A. Risk factors for depression and anxiety in abstainers, moderate drinkers and heavy drinkers. Addiction 2000; 95: 1833-45.

16. Australian Institute of Health and Welfare (AIHW). 2007 National Drug Strategy Household Survey: First Results. Canberra: Australian Institute of Health and Welfare; 2008.

17. National Health and Medical Research Council (NHMRC). Australian Guidelines to Reduce Health Risks from Drinking Alcohol. Canberra: Australian National Health and Medical Research Council; 2009.

18. Fillmore K. M., Kerr W. C., Stockwell T., Chikritzhs T., Bostrom A. Moderate alcohol use and reduced mortality risk: systematic error in prospective studies. Addict Res Theory 2006; 14: 101-32.

19. Liang W., Chikritzhs T., Fillmore K. M. Mental disorders the missing piece? Addiction 2010; 105: 1497-8

20. Niti M., Ng T.-P., Kua E. H., Ho R. C. M., Tan C. H. Depression and chronic medical illnesses in Asian older adults: the role of subjective health and functional status. Int J Geriatr Psychiatry 2007; 22: 1087-94.

21. Ramkumar A., Quah J. L., Wong T., Yeo L. S., Nieh C. C., Shankar A. et al. Self-rated health, associated factors and diseases: a community-based cross-sectional study of Singaporean adults aged 40 years and above. Ann Acad Med Singapore 2009; 38: 606-7.

22. Kerr W. C., Fillmore K. M., Bostrom A. Stability of alcohol consumption over time: evidence from three longitudinal surveys from the United States. J Stud Alcohol 2002; 63: 325-33.

23. Kavey R.-E. W., Daniels S. R., Lauer R. M., Atkins D. L., Hayman L. L., Taubert, K. American heart association guidelines for primary prevention of atherosclerotic cardiovascular disease beginning in childhood. Circulation 2003; 107: 1562-6.

24. Shaw J. E., Chisholm D. J. 1: Epidemiology and prevention of type 2 diabetes and the metabolic syndrome. Med J Aust 2003; 179: 379-83.

25. Pearson T. A., Blair S. N., Daniels S. R., Eckel R. H., Fair J. M., Fortmann S. P. et al. AHA guidelines for primary prevention of cardiovascular disease and stroke: 2002 update: consensus panel guide to comprehensive risk reduction for adult 
patients without coronary or other atherosclerotic vascular diseases. Circulation 2002; 106: 388-91.

26. Prince M., Patel V., Saxena S., Maj M., Maselko J., Phillips M. R. et al. No health without mental health. Lancet 2007; 370: 859-77.

27. Hollis S., Campbell F. What is meant by intention to treat analysis? Survey of published randomised controlled trials. BMJ 1999; 319: 670-4.

28. Montori V. M., Guyatt G. H. Intention-to-treat principle. Can Med Assoc J 2001; 165: 1339-41.

29. Stockwell T., Donath S., Cooper-Stanbury M., Chikritzhs T., Catalano P., Mateo C. Under-reporting of alcohol consumption in household surveys: a comparison of quantityfrequency, graduated-frequency and recent recall. Addiction 2004; 99: 1024-33.

\section{Supporting information}

Additional Supporting Information may be found in the online version of this article:
Table S1 Estimations on key dependent variables by samples from combined sample, sample from 2007 survey and sample from 2004 survey.

Table S2 Estimations from full model excluding subjects with missing values and estimations from full sample controlling for diagnosis of disease/general health status, sex and age.

Table S3 Comparison results from logistic regression model, Poisson model and multinominal logistic regression model.

Please note: Wiley-Blackwell are not responsible for the content or functionality of any supporting materials supplied by the authors. Any queries (other than missing material) should be directed to the corresponding author for the article. 